\title{
MATHEMATICS AND STRING THEORY
}

\section{Principal Investigator: Shing-Tung Yau Department of Mathematics \\ Harvard University \\ Cambridge, MA 02138}

\section{FINAL REPORT ON DOE GRANT DE-FG02-88ER25065}

The Principal Investigator wrote several papers covering the following areas:

The continuation of collaboration with Liu and Lian on calculation of II A model, opens up possibility to understand calculations for higher genus curves also. A lot of detail calculations based on the idea of Liu-Lian-Yau method was carried out by Lian-Liu-Yau. It provides evidence that the method is powerful to calculate GW invariants in many cases. Local mirror symmetry is also worked out by Chiang, Klemm, Zaslow and the principal investigator. It is consistant with what is predicted by physics intuition. Many works were carried out on pushing forward the ideas of Strominger-Yau-Zaslow 's geometric version of mirror symmetry in terms of special Lagragian torus fibration. Several papers were carried out with Zaslow, Leung and others on understanding such duality. It fits beautifully with the predictions. The ideas are still being studied.

\section{LIST OF PUBLICATIONS}

DOE Patent Clearance Granted

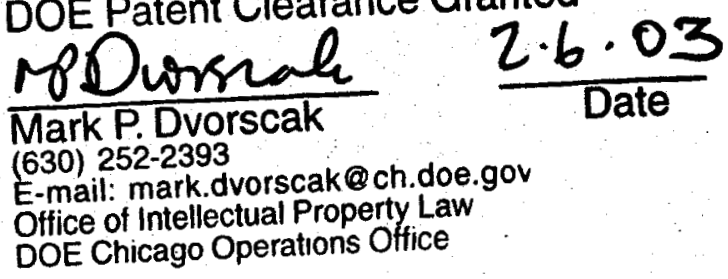




\section{DISCLAIMER}

This report was prepared as an account of work sponsored by an agency of the United States Government. Neither the United States Government nor any agency thereof, nor any of their employees, makes any warranty, express or implied, or assumes any legal liability or responsibility for the accuracy, completeness, or usefulness of any information, apparatus, product, or process diselosed, or represents that its use would not infringe privately owned rights. Reference herein to any specific commercial product, process, or service by trade name, trademark, manufacturer, or otherwise does not necessarily constitute or imply its endorsement, recommendation, or favoring by the United States Government or any agency thereof. The views and opinions of authors expressed herein do not necessarily state or reflect those of the United States Governmeat or any agency thereof. 


\section{DISCLAIMER}

Portions of this document may be illegible in electronic image products. Images are produced from the best available original document. 
Naichung Conan Leung, Shing-Tung Yau, Eric Zaslow; "From Special Lagrangian to HermitianYang-Mills via Fourier-Mukai Transform" Adv.Theor.Math.Phys. 4 (2002) 1319-1341 math.DG/0005118

Sergei Gukov, Shing-Tung Yau, Eric Zaslow; "Duality and Fibrations on G_2 Manifolds" hep-th/0203217

T.-M. Chiang, A. Klemm, S.-T. Yau, E. Zaslow; "Local Mirror Symmetry: Calculations and Interpretations" Adv.Theor.Math.Phys. 3 (1999) 495-565

hep-th/9903053

Andrew Strominger, Shing-Tung Yau, Eric Zaslow; "Mirror Symmetry is T-Duality" Nucl.Phys. B479 (1996) 243-259

hep-th/9606040

Shing-Tung Yau, Eric Zaslow; "BPS States, String Duality, and Nodal Curves on K3" Nucl.Phys. B471 (1996) 503-512

hep-th/9512121

Bjorn Andreas, Gottfried Curio, Daniel Hernandez Ruiperez, Shing-Tung Yau; "Fibrewise TDuality for D-Branes on Elliptic Calabi-Yau" JHEP 0103 (2001) 020

hep-th/0101129

Bjorn Andreas, Gottfried Curio, Daniel Hernandez Ruiperez, Shing-Tung Yau; "Fourier-Mukai Transform and Mirror Symmetry for D-Branes on Elliptic Calabi-Yau"

math.AG/0012196

Bong H. Lian (Brandeis University), Chien-Hao Liu (Harvard University), Kefeng Liu (University of California at Los Angelas), Shing-Tung Yau (Harvard University); "The $\$ S^{\wedge} 1 \$$ fixed points in Quot-schemes and mirror principle computations"

math.AG/0111256

Yi Hu (U. Texas at Arlington), Chien-Hao Liu, Shing-Tung Yau (Harvard University); "Toric morphisms and fibrations of toric Calabi-Yau hypersurfaces"

math.AG/0010082

Bong H. Lian (Brandeis University), Chien-Hao Liu, Shing-Tung Yau (Harvard University); "A reconstruction of Euler data"

math.AG/0003071

Chien-Hao Liu, Shing-Tung Yau (Harvard University); "On the Splitting Type of an Equivariant Vector Bundle over a Toric Manifold"

math.AG/0002031

B. Lian, K. Liu, S.T. Yau; "Mirror Principle I"

alg-geom/9712011 
Shinobu Hosono, Bong H. Lian, Keiji Oguiso, Shing-Tung Yau; "Autoequivalences of Derived Category of A K3 Surface and Monodromy Transformations" math.AG/0201047

Bong H. Lian, Andrey Todorov, Shing-Tung Yau; "Maximal Unipotent Monodromy for Complete Intersection CY Manifolds" math.AG/0008061

A. Klemm, B. Lian, S.-S. Roan, S.-T. Yau; "Calabi-Yau fourfolds for M- and F-Theory compactifications" Nucl.Phys. B518 (1998) 515-574

hep-th/9701023

S.Hosono, B.H.Lian, S.-T.Yau; "Calabi-Yau Varieties and Pencils of K3 Surfaces" alg-geom $/ 9603020$

S.Hosono, B.H.Lian, S.-T.Yau; "Maximal Degeneracy Points of GKZ Systems" alg-geom/9603014

S.Hosono, B.H.Lian, S.-T.Yau; "GKZ-Generalized Hypergeometric Systems in Mirror Symmetry of Calabi-Yau Hypersurfaces" Commun.Math.Phys. 182 (1996) 535-578 alg-geom/9511001

Bong H. Lian, Shing-Tung Yau; "Mirror Maps, Modular Relations and Hypergeometric Series. II" Nucl.Phys.Proc.Suppl. 46 (1996) 248-262 hep-th/9507153

Bong H. Lian, Shing-Tung Yau; "Mirror Maps, Modular Relations and Hypergeometric Series I" hep-th/9507151

Bong H. Lian, Shing-Tung Yau; "Arithmetic Properties of Mirror Map and Quantum Coupling". Commun.Math.Phys. 176 (1996) 163-192

hep-th/9411234

A. Klemm, B.H. Lian, S.S. Roan, S.-T. Yau; "A Note on ODEs from Mirror Symmetry" hep-th/9407192 